\title{
Body Area Network for Monitoring Autonomic Nervous System Responses
}

\author{
L. Brown, B. Grundlehner, J. van de Molengraft, J. Penders, B. Gyselinckx \\ Holst Centre \\ Eindhoven, The Netherlands \\ lindsay.brown@imec-nl.nl
}

\begin{abstract}
A body area network (BAN) for monitoring the autonomic nervous system responses is reported. The BAN is based on the Human++ UniNode, a small, low power generic wireless sensor node. Physiological signals are monitored using specifically designed ultra low power sensor front ends connected to the UniNodes. Two UniNodes compose the body area network, one on a chest belt to record ECG and respiration, the other on a wrist sensor to record skin conductance and skin temperature. Small, lightweight and low power body area network platform, this platform BAN platform paves the way towards ambulatory, continuous monitoring of autonomic responses in everyday applications.
\end{abstract}

\section{INTRODUCTION}

Body area networks (BAN) are becoming increasingly popular for providing ambulatory pervasive health monitoring. They lead the way in providing the user with health, lifestyle and entertainment functions, while maintaining user mobility and comfort. Body area networks have typically been designed for a specific application, with a sensor node developed and used to monitor one or two specific parameters. However, the nature of body area networks allows for improved configuration flexibility through the use of interchangeable sensors, so that each network can be made application specific while re-using a generic platform.

At the same time, pervasive health monitoring is becoming increasingly popular. Applying pervasive tools for the monitoring of autonomic nervous system (ANS) responses during stress and emotions, adds an extra dimension to the more classical physical health monitoring. A few groups have developed portable monitoring platforms with the goal to study emotions, such as the MIT Media Lab [1], and Fraunhofer Institute [2].

Building on previous work on body area networks [3], [4], this paper presents a body area network platform for monitoring ANS responses, with a focus on low-power consumption and comfort of use. The system is evaluated for recognition of 3 discrete emotions, suggesting how it may enable real-time, continuous monitoring of an individual's emotions.

\section{BODY AREA NETWORK FOR ANS RESPONSE MONITORING}

Typically, wireless sensor nodes consist of one or more sensor front-end circuits, a microcontroller to sample and process data, a power supply and a radio to transmit the data. While the type of sensor might differ from sensor node to sensor node, the microcontrollers and radios are normally the same across a single wireless sensor network. Hardware systems have in the past usually been developed for specific applications, with reduced flexibility for adding or subtracting sensors. By using a generic sensor node platform, sensors can be interchanged easily based on the demands of the target application.

\section{A. Human++ UniNode}

The Human++ UniNode is a small and generic wireless sensor node developed to provide a default platform to which different sensors can interface.

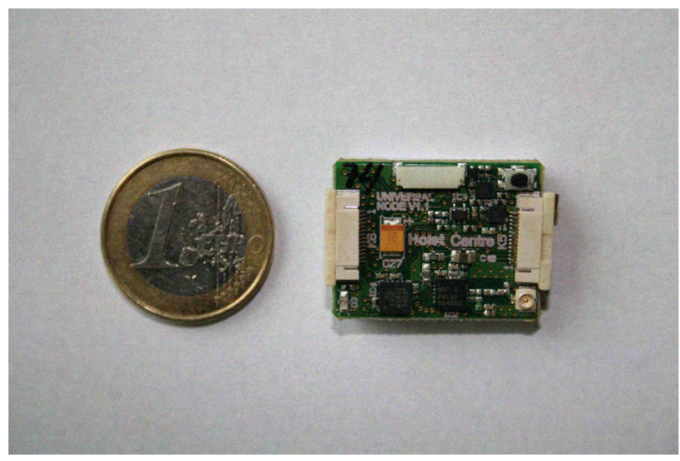

Fig. 1. The Human++ UniNode

The UniNode contains three default blocks: a radio, microcontroller and a power circuit. The UniNode uses a Texas Instruments MSP430 series microcontroller, Nordic nRF24L01 $2.4 \mathrm{GHz}$ radio, $50 \mathrm{Ohm}$ antenna, and is powered by a $165 \mathrm{mAh}$ lithium-ion battery. The total size of the UniNode, including battery, is $20 \times 29 \times 9 \mathrm{~mm}$.

Each UniNode module handles 2 data channels. The two UniNodes in the BAN for ANS monitoring communicate with a central receiver node in a star network using a static TDMA protocol. The TDMA cycle is $33 \mathrm{~Hz}$, with 6 samples per cycle per channel sent, the total sampling rate is $198 \mathrm{~Hz}$. Data can be transmitted to a computer for real time display, storage and computation, or to a datalogger for storage and later downloading.

\section{B. Sensors for monitoring ANS responses}

Two sensor front-ends have been designed for the read-out and conditioning of ECG, respiration, skin conductance and skin temperature signals. 
The ECG and respiration measurements use the ExG sensor front-end readout circuit, which relies on a proprietary single channel ASIC for biopotential read-out [5]. The ASIC is an ultra low power and high performance front end for bio-potential applictions. It consists of AC coupled chopped instrumentation amplifier, a spike filter, and amplification stage with constant gain, and a variable gain amplifier stage. The variable gain amplifier can be used to electronically adjust the gain of the readout for varying needs of EEG, ECG and EMG applications. A piezoelectric film sensor from SleepSense is used to measure respiration. With the output of the piezoelectric material of the order of microvolts, a second ExG sensor readout is used to amplify and filter the signal before it is passed into the microcontroller ADC.

Skin conductance is measured between two locations on the hand, typically between the base of two fingers. The readout design is based on the design of the MIT Handwave wireless skin conductance sensor circuit [1], where a constant voltage of $0.5 \mathrm{~V} \mathrm{DC}$ is applied, and the change in current is measured. For skin temperature, the Melexis MLX90614 plug-and-play digital infrared thermometer module is connected to the same sensor board. The temperature sensor achieves high resolution of $0.02^{\circ}$ to an accuracy of $0.05^{\circ}$ over the range $0^{\circ}$ to $50^{\circ}$.

\section{Body Area Network for monitoring ANS responses}

These four sensors are integrated into a wearable, wireless platform for monitoring physiological signals associated with ANS responses. The ECG and respiration sensors were connected to one Human++ UniNode and integrated into a chest belt (Chest node), while the skin conductance and skin temperature sensors were connected to a second Human++ UniNode and integrated into a wrist band (Wrist node). The integrated platform is shown in Fig. 2. Fig. 3 illustrates typical signals as collected using the system in an ambulatory environment, with the subject giving a speech.

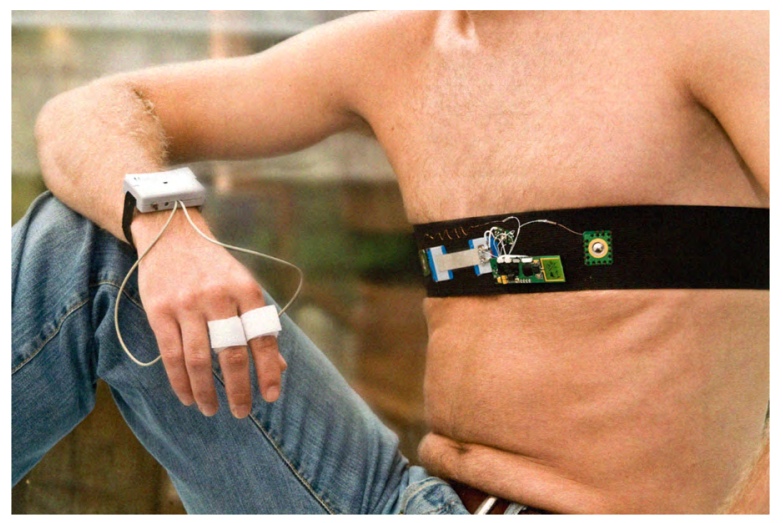

Fig. 2. Wireless Emotion Monitoring Body Area Network

Each ExG sensor board used for ECG and respiration sensor is $20 \times 22 \times 4 \mathrm{~mm} 3$, while the sensor board used by the skin conductance and skin temperature sensor is $20 \times 25 \times 5$ mm3. The combination of these sensors with the Human++

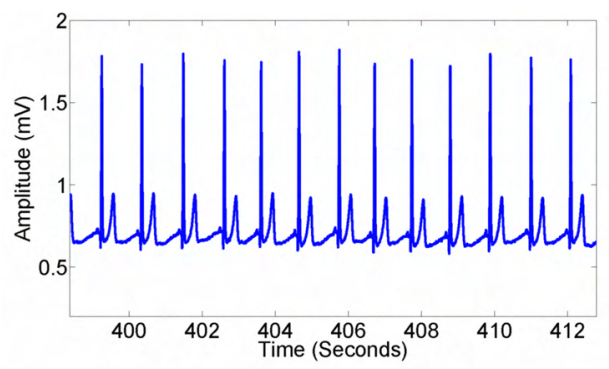

(a) ECG

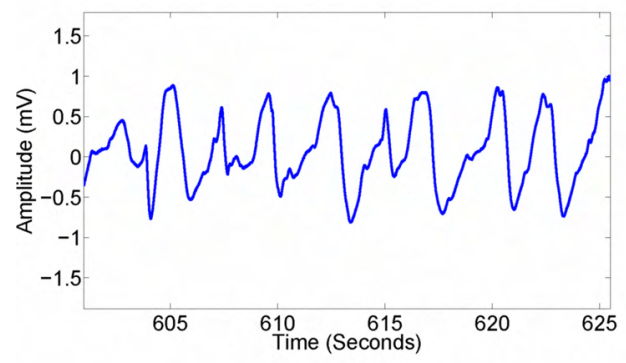

(b) Respiration

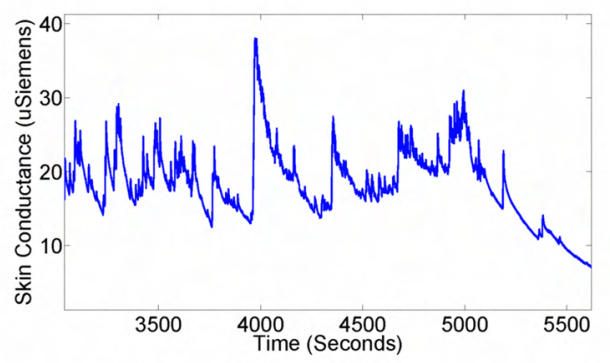

(c) Skin Conductance

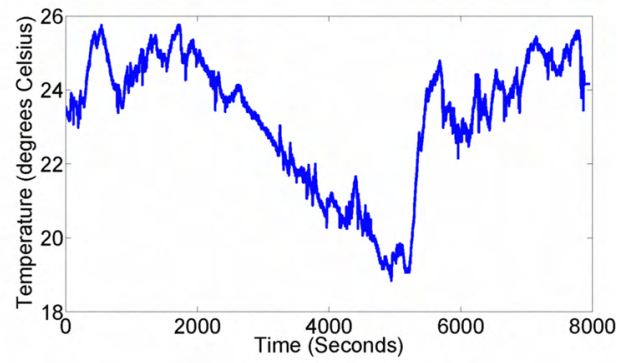

(d) Skin Temperature

Fig. 3. ECG, Respiration, Skin Conductance and Skin Temperature recorded using the Human++ BAN for ANS monitoring.

UniNode provides very small and power efficient sensors for the body area network. The chest node consumes 2.6 $\mathrm{mA}$ in full operation, while the wrist node consumes $4 \mathrm{~mA}$, resulting in an approximative battery lifetime of 63 hours and 41 hours respectively. The high power consumption of the skin conductance / skin temperator sensor node is due to the 1-2 $\mathrm{mA}$ current drawn by the continuous operation of the infrared temperature sensor. This can be reduced by duty cycling the temperature measurement. Both sensor nodes have a resolution 
of 12 bits, sampling frequency of $198 \mathrm{~Hz}$ and a range of 10 meters. Filtering was applied to the ECG, respiration and skin conductance signals before analog to digital conversion. The low-pass cutoff frequencies were $30 \mathrm{~Hz}, 30 \mathrm{~Hz}$ and $45.2 \mathrm{~Hz}$ respectively.

\section{Platform performances}

Table I summarises the key performance indicators of the generic Human++ UniNode wireless body area network platform.

TABLE I

Performance Characteristics

\begin{tabular}{cc}
\hline \hline Power UniNode (without sensors) at 3 V & $7.5 \mathrm{~mW}$ \\
Power Chest node at 3 V & $7.8 \mathrm{~mW}$ \\
Power Wrist node at 3 V & $12 \mathrm{~mW}$ \\
Resolution & $12 \mathrm{bits}$ \\
Sampling Frequency & $198 \mathrm{~Hz}$ \\
RF Band & $2.4 \mathrm{GHz} \mathrm{ISM}$ Band \\
Battery capacity & $165 \mathrm{mAh}$ \\
Transmission rate & $1 \mathrm{Mbps}$ \\
Range & $10 \mathrm{~m}$ \\
\hline \hline
\end{tabular}

\section{EVALUATION STUDY}

To pervasively monitor emotional state, the ANS resposes during emotional stimulation are commonly measured. In this context, the BAN for monitoring ANS responses was evaluated as an enabling technology for pervasively monitoring emotional states. 10 subjects (mean age 29.3, 3 females, 7 males) watched 5 emotionally stimulating film clips selected to elicit sadness, happiness, fear, disgust and neutrality [6]. During the stimulation, the subjects wore the BAN to caputre the ANS responses. Each film clip was between 110 seconds and 430 seconds, with clips separated by a 180 second period of calming music. At the end of each clip, the subjects filled a self-report questionnaire rating the most intense emotion felt (selecting from anger, disgust, fear, sadness, happiness and neutral) and the intensity (scale 1 , not intense to 5 , very intense). The 5 film clips were then further grouped into 3 categories for simplicity. The categories used were fear and disgust, happiness and neutral, and sadness. The 4 physiological signals were then analysed offline.

Although four physiological signals were recorded, only heart rate and skin conductance related features based on previous studies on emotion recognition [7], were used. These were: mean, standard deviation, difference between maximum and minimum value, difference between last and first value and the mean of the absolute second difference signal of both heart rate and skin conductance signals, heart rate variability, the number of peaks per second in the skin conductance and the average amplitude of the peaks in the skin conductance. All statistical features are computed within a sliding rectangular window of one second, and after normalization [8]. These features were mapped to 2 axis using Fisher Mapping. Linear Discriminant Classification was finally used to classify the data with accuracy of $64 \%$, computed using (leave-one-out) crossvalidation on the data-set. This result was expected and inline with previous work [2], [7], [8], showing the effectiveness of this platform in the context of pervasive monitoring of ANS responses during emotional stimulation.

The BAN presented here is shown to work effectively to monitor the ANS responses of ECG, respiration, skin conductance and skin temperature during emotional stimulation. Using only heart rate and skin conductance features, linear discriminant classification was able to correctly classify the emotional state with $64 \%$ accuracy.

Further improvements can be made to increase the sensor integration and wearability of the sensors, to further enhance the usefulness in an ambulatory research environment. In doing so, the body area network for monitoring ANS responses can be used as an enabling technology platform for research in the field of psychophysiological research and pervasive health monitoring.

\section{CONCLUSIONS}

This paper presented a modular low-power body area network for monitoring ANS responses. The system relies on a generic wireless sensor node, the Human++ UniNode, to which different sensor front-end readout circuits can be connected. Here, ECG, respiration, skin conductance and skin temperature sensors have been connected to two UniNodes and integrated into wearable sensors suitable for ambulatory, continuous monitoring ANS responses to external stimuli. The system was tested in a controlled environment for the monitoring of discrete emotions, with $64 \%$ accuracy in classifying emotion state. This paper suggests that, when applied to monitoring ANS and other mental health related signals, comfortable and low-power BAN technology may enable realtime ambulatory emotion monitoring in the future.

\section{REFERENCES}

[1] M. Strauss, "Handwave: Design and manufacture of a wearable wireless skin conductance sensor and housing," Master's thesis, MIT, June 2005.

[2] C. Peter, E. Ebert, and H. Beikirch, "A wearable multi-sensor system for mobile acquisition of emotion-related physiological data," in Proceedings of the 1st International Conference on Affective Computing and Intelligent Interaction, 2005.

[3] N. de Vicq, F. Robert, J. Penders, B. Gyselinckx, and T. Torfs, "Wireless body area network for sleep staging," in Proc. Int. Conf. on Biological Circuits and Systems, 2007.

[4] J. Penders, B. Gyselinckx, N. de Vicq, and T. Torfs, "Body area networks for multi-modal biomedical monitoring," in pHealth, 2007.

[5] R. F. Yazicioglu, R. F. Yazicioglu, P. Merken, R. Puers, and C. Van Hoof, "A $60 \mu \mathrm{W} 60 \mathrm{nV} / \sqrt{ } \mathrm{Hz}$ readout front-end for portable biopotential acquisition systems," IEEE Journal of Solid-State Circuits, vol. 42, no. 5 , pp. 1100-1110, 2007.

[6] J. Gross and R. Levenson, "Emotion elicitation using films," Cognition and Emotion, vol. 9, no. 1, pp. 87-108, 1995.

[7] C. Collet, E. Vernet-Maury, G. Delhomme, and A. Dittmar, "Autonomic nervous system response patterns specificity to basic emotions." J Auton Nerv Syst, vol. 62, no. 1-2, pp. 45-57, Jan 1997.

[8] R. Picard, E. Vyzas, and J. Healey, "Toward machine emotional intelligence: analysis of affective physiological state," IEEE Transactions on Pattern Analysis and Machine Intelligence, vol. 23, no. 10, pp. 11751191, 2001. 